\title{
The hydrodynamics of a recirculating (O-tube) flume
}

\author{
H. Mohr \& S. Draper \\ Centre for Offshore Foundation Systems \& School of Civil, Environmental and Mining Engineering \\ The University of Western Australia, Perth, Australia
}

D.J. White

Centre for Offshore Foundation Systems \& Shell EMI Chair of Offshore Engineering

The University of Western Australia, Perth, Australia

L. Cheng, H. An \& Q. Zhang

School of Civil, Environmental and Mining Engineering

The University of Western Australia, Perth, Australia

\begin{abstract}
An O-tube flume is a horizontal closed-circuit flume that can be driven by an inline impellertype pump to produce steady and/or oscillatory flow over a mobile seabed. An O-tube has the ability to reproduce large combined wave and current conditions near the seabed, typical of (cyclonic) storm conditions. In this paper, we investigate the hydrodynamics of an O-tube and show applications of this technology. First, we derive a dynamic equation to explain the coupling between pressure and flow rate within the O-tube. This result can be thought of as an extension of the classical hydrodynamic equations developed for U-tubes and allows for improved control of the flow within the O-tube by providing a prediction of the non-linear interaction between steady flow (i.e. currents) and unsteady flow (i.e. waves). We demonstrate this improved control by comparing measurements of flow rate taken from an O-tube with the dynamic equation. Secondly, we present velocity measurements to give a detailed description of the flow field within the O-tube, including mean flow profiles and seabed shear stress. Finally, we conclude the paper by providing two example applications of the facility to study sediment transport and scour.
\end{abstract}

\section{INTRODUCTION}

Many different flumes have been developed to study scour and morphodynamics at the laboratory scale in steady and/or oscillatory flows. These include (i) open top flumes, which can be configured with a wave paddle, (ii) stationary or unidirectional flumes fitted with an oscillating trolley (e.g. Bagnold and Taylor (1946); Hammond and Collins (1979)) and (iii) enclosed flumes (or oscillating water tunnels) in which water may be allowed to oscillate freely in a vertical U-tube or is driven by a range of piston type arrangements (such as the large oscillating tunnel at Delft Hydraulics, the Aberdeen Oscillating tunnel, and the Pulsating water tunnel; Ribberink and Al-Salem (1994); O'Donoghue and Wright (2004); Murray (1992)).

As is to be expected, each of these different flumes has both advantages and disadvantages. Open top flumes with a wave paddle, for example, reproduce the kinematics of orbital wave motion, but they are limited to wave velocities and periods below that which lead to wave breaking and to significant wave reflection within the flume. In comparison, trolleys in stationary and unidirectional flumes are not limited by wave breaking and reflection, but they do not reproduce the same kinematics as a progressive wave and can be inaccurate if the inertia force is large (Davies \& Wilkinson 1978). U-tubes are not limited by inertia force and, as is typical for all oscillating water columns that do not feature a free water surface, they are not limited by wave breaking criteria. However, they are usually limited to the resonant period of the water mass and are difficult to adapt to incorporate steady current velocities. Piston driven water tunnels can be adapted to incorporate steady current (for example the Tokyo University Water Tunnel and the HR Wallingford Pulsating Water Tunnel; Ahmed (2002); Murray (1992)), however, the oscillatory flow amplitude in these facilities is limited by the length of the piston stroke.

In this paper, we focus on a new recirculating flume concept known as an O-tube. This flume comprises 
a horizontal fully enclosed circulating water channel, which includes a rectangular test section and an impeller-type pump driven by a motor (see An et al. (2013); Figure 1). This arrangement has the relative advantages that (i) currents can be introduced easily, and (ii) wave velocities are limited only by the pump characteristics and not by wave breaking, resonance of the water mass or the stroke of a piston (see Section 4). These advantages reflect the motivation for the development of the O-tube concept, which is to investigate stability of subsea pipelines on mobile seabeds in extreme (cyclonic) storm wave and current conditions (Cheng, White, Palmer, Jas, Czajko, Fogliani, Fricke, \& An 2010). However, like all enclosed flumes, the O-tube is restricted to near-seabed scenarios in which the vertical motion of the fluid due to waves is not significant. Additionally, it cannot reproduce the same pressure variation and orbital flow kinematics experienced near the seabed under a progressive wave. This difference can be important for reproducing and interpreting physical phenomena such as seabed liquefaction.

In light of this limitation, the primary aim of this paper is to provide a theoretical description of the hydrodynamics within an O-tube which can be seen as an extension of the classical approach that Streeter (1958) presented for U-tubes. In particular, the derived relationship between the flow rate and pressure within the tube is important for optimising the control of the flume under combined wave and current conditions, and for interpretation of experiments including the observed phenomena such as sediment transport and seabed liquefaction. A second aim of this paper is to give a detailed description of velocity measurements within the working section of an $\mathrm{O}$ tube. These measurements summarise mean flow and seabed shear stress. The paper then concludes by describing recent experiments undertaken in an O-tube. A thorough description of the technology and control of an existing O-tube flume constructed at the University of Western Australia (and described briefly in Section 2) is presented by An et al. (2013).

\section{O-TUBE FACILITIES}

Two O-tubes have been constructed at the University of Western Australia. The first is a Large O-tube (LOT) with an overall length of $24 \mathrm{~m}$, breadth $7.8 \mathrm{~m}$ and a working section of $17.4 \mathrm{~m}$ (length) $\times 1.4 \mathrm{~m}$ $($ high $) \times 1.0 \mathrm{~m}$ (wide). This O-tube can achieve a maximum steady current velocity of up to $3 \mathrm{~m} / \mathrm{s}$ and an oscillatory flow velocity of up to $1 \mathrm{~m} / \mathrm{s}-2.5 \mathrm{~m} / \mathrm{s}$, respectively, for wave periods of 5-13 s (see An et al. (2013), for further details). The LOT can be used for 1:1 scale experimental studies of subsea pipelines in, for example, cyclonic conditions representing the North West Shelf offshore Australia.

The second O-tube is approximately 5 times smaller than the LOT, and is termed the Mini O-tube

\begin{tabular}{ll}
\hline Specification & Quantity \\
\hline Max. steady velocity & $1.5 \mathrm{~m} / \mathrm{s}$ \\
& \\
Flow amplitude, & $0.5 \mathrm{~m} / \mathrm{s}, 2.5 \mathrm{~s}, 1.3 \mathrm{kPa} / \mathrm{m}$ \\
period, & $0.9 \mathrm{~m} / \mathrm{s}, 5 \mathrm{~s}, 1.1 \mathrm{kPa} / \mathrm{m}$ \\
pressure gradient & $1.1 \mathrm{~m} / \mathrm{s}, 7.5 \mathrm{~s}, 0.9 \mathrm{kPa} / \mathrm{m}$ \\
& $1.2 \mathrm{~m} / \mathrm{s}, 10 \mathrm{~s}, 0.8 \mathrm{kPa} / \mathrm{m}$ \\
& $1.5 \mathrm{~m} / \mathrm{s}, 12.5 \mathrm{~s}, 0.8 \mathrm{kPa} / \mathrm{m}$ \\
\hline
\end{tabular}

Table 1: Summary of MOT characteristics.

(MOT); see Figure 1. The MOT has a working test cross-section $1.8 \mathrm{~m}$ (length) $\times 0.3 \mathrm{~m}$ (high) $\times 0.2 \mathrm{~m}$ (wide) and measures $6.4 \mathrm{~m}$ long. Soil is placed in a $0.1 \mathrm{~m}$ deep by $0.2 \mathrm{~m}$ wide cavity at the bottom of the working section. Compared with the LOT, the MOT has the advantage that it requires less sediment to fill and nourish the working section (so that small scale tests and erosion testing can be undertaken with prototype sediment) and it allows for testing at comparatively reduced scale (so as to examine scale effects). The experimental results discussed in this paper are from the MOT.

The MOT comprises of a motor-impeller system, $150 \mathrm{~mm}$ diameter PVC pipe sections, two $200 \mathrm{~mm}$ long flow straighteners at each end of the test section (with internal honeycomb diameter of $25 \mathrm{~mm}$ ), two vertical tubes to bleed air from the system at each end of the test section and one transparent working section. A summary of the dimensions and other specifications of the MOT are given in Table 1. Although preliminary CFD modelling was undertaken to select the overall dimensions of the LOT, the dimensions of the MOT were not optimised in any quantitative manner since it was originally designed merely as a proofof-concept for the LOT.

\section{HYDRODYNAMICS OF AN O-TUBE}

In contrast to well-established methods for predicting U-tube flow (e.g. Streeter (1958); Richardson (1967); Biery (1969)), at present there is no hydrodynamic theory to describe bulk flow within an O-tube flume. In this section, we develop the equivalent theory for an O-tube starting from the most basic dynamic equation describing flow within the O-tube (or any enclosed water flume generally)

$$
\frac{\partial u}{\partial t}+\frac{1}{\rho} \frac{\partial p}{\partial x}+u \frac{\partial u}{\partial x}=-F
$$

where $\rho$ is fluid density, $u(x, t)$ and $p(x, t)$ are the (bulk) velocity and pressure, averaged over the crosssectional area of the O-tube, $x$ is the position along the O-tube (see Figure 1) and $t$ is time. The term, $F$, on the right hand side of (1) represents O-tube wall resistance per unit mass of fluid in the O-tube. Continuity also implies that the volumetric flow rate within 

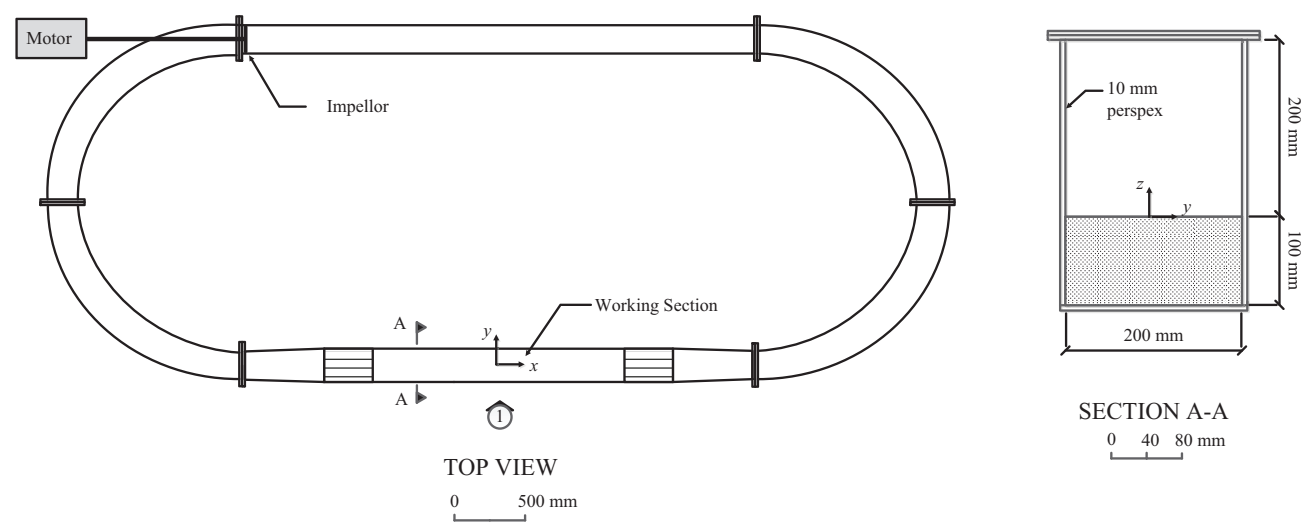

SECTION A-A $\stackrel{0}{4} \quad 40 \quad 80 \mathrm{~mm}$

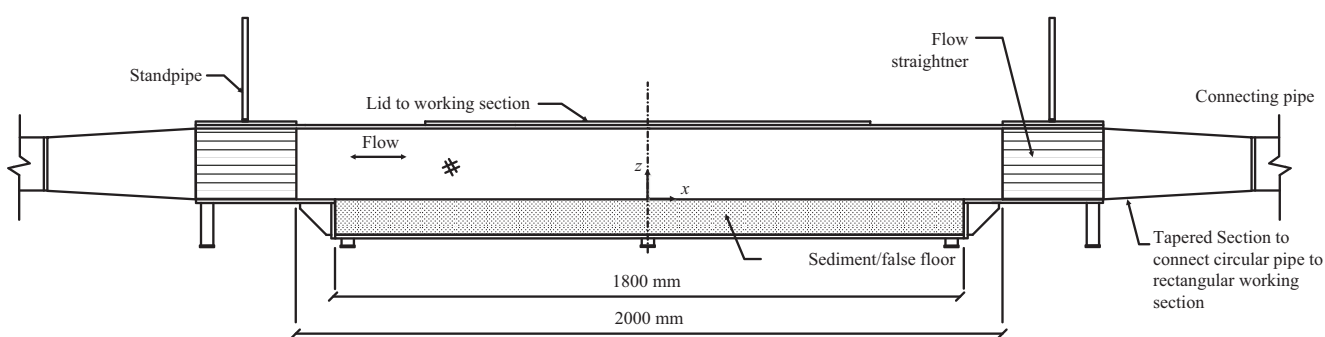

ELEVATION 1: WORKING SECTION

$\begin{array}{lll}0 & 100 & 200 \mathrm{~mm}\end{array}$

Figure 1: Detailed dimensions of the Mini O-tube (MOT). Coordinate system also shown.

the O-tube (again as in any enclosed water flume) can only be a function of time; i.e.,

$Q(t)=u(x, t) A(x)$,

where $Q$ is the volumetric flow rate and $A(x)$ is the cross-sectional area of the tube. Unlike the flow rate, the average velocity will vary around the O-tube (for example, due to variations in cross-sectional area at the entrance and exit to the O-tube working section) and is, therefore, not independent of position. Substituting (2) into (1) leads to

$\frac{1}{A} \frac{\partial Q}{\partial t}+\frac{1}{\rho} \frac{\partial p}{\partial x}+\frac{1}{2} \frac{\partial}{\partial x}\left(\frac{Q^{2}}{A^{2}}\right)=-F$

This can now be integrated around the O-tube, either side of the propeller, to give

$\Delta p=a_{1} \frac{\mathrm{d} Q}{\mathrm{~d} t}+\rho \int_{0}^{L} F \mathrm{~d} x$

where $a_{1}=\rho \int_{0}^{L} A^{-1} d x$ is a constant related to the mass of water in the O-tube (and can, therefore, be obtained from calibration as shown later), while $\Delta p(t)=p(0, t)-p(L, t)$ is equal to the driving pressure from the impeller. Note that the third term on the left hand side in (3) disappears because the integral is closed around the O-tube.

Following the usual assumptions of flow in pipe networks the integral on the right hand side of (4), which describes the resistance along the pipe, can be rewritten as $\rho \int_{0}^{L} F \mathrm{~d} x=f \int_{0}^{L} \frac{\rho u^{2}}{2 D_{H}} \mathrm{~d} x+\sum k_{i} \frac{\rho u_{i}^{2}}{2}$,

where $f$ is the pipe friction factor (which can be obtained from the well-known Moody diagram; Moody (1944)), $D_{H}$ is the hydraulic diameter, and $u_{i}$ and $k_{i}$ are the local average velocity and head loss coefficient associated with the $i$ th bend, expansion, contraction etc. along the pipe. Allowing for flow in both directions, (5) can, therefore, be rewritten in terms of the flow rate as

$\rho \int_{0}^{L} F \mathrm{~d} x=a_{2} Q|Q|$,

with

$a_{2}=\int_{0}^{L} \frac{\rho f}{2 A^{2} D_{H}} \mathrm{~d} x+\sum \frac{\rho k_{i}}{2 A_{i}^{2}}$,

where $A_{i}$ is the cross-sectional area at the locations of head loss. Importantly, like the parameter $a_{1}$, the parameter $a_{2}$ in (6a) should also be a constant value for a given O-tube, and could, therefore, be obtained simply from calibration (explained further below). Using (6a), (4) now becomes

$\Delta p=a_{1} \frac{\mathrm{d} Q}{\mathrm{~d} t}+a_{2} Q|Q|$

so that the flow rate is related to the pressure via a non-linear first order differential equation. The form 
of (7) illustrates that the pressure supplied by the impeller is balanced by the acceleration of fluid within the pipe (the first term on the right hand side) and the drag losses in the pipe (the second term on the right hand side).

In practice, the pressure (or head) delivered by an impeller is typically proportional to the square of its rotation speed (via the Affinity Laws) and so, preserving signs, (7) can be rewritten as

$$
N|N|=a_{1}^{\prime} \frac{\mathrm{d} Q}{\mathrm{~d} t}+a_{2}^{\prime} Q|Q|,
$$

where $N$ is the rotation speed of the impeller (typically measured in revolutions per minute (rpm)) and the coefficients are $a_{1}^{\prime}=a_{1} / a_{3}$ and $a_{2}^{\prime}=a_{2} / a_{3}$, where $a_{3}\left(1.6 \times 10^{-3} \mathrm{~Pa} / \mathrm{rpm}\right)$ is a constant of proportionality between the pressure and the square of the rotation speed of the impeller (and can be obtained from, for example, measurements such as those reported later in Figure 6.

Equation (8) is the final governing equation which relates the impeller speed to the flow rate in the $\mathrm{O}$ tube. In the following sections, we consider some simple solutions to (8) and compare the results with measurement from the MOT. These solutions ultimately allow for both constants $a_{1}^{\prime}$ and $a_{2}^{\prime}$ to be obtained empirically so that (8) can then be used to predict the flow rate produced in the O-tube for any chosen impeller rotation speeds. It would be possible to nondimensionalise Equation (8), but dimensions are retained herein to allow for easy interpretation of the coefficients $a_{1}^{\prime}$ and $a_{2}^{\prime}$.

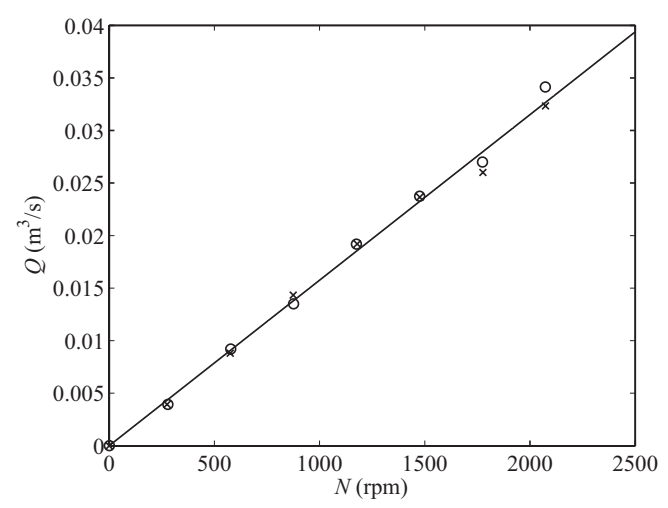

Figure 2: Comparison of measured flow rate with steady impeller rotation speed $N$. The best fit trend line suggests that $a_{2}^{\prime}=4.14 \times 10^{9} \mathrm{rpm}^{2} \mathrm{~s}^{2} / \mathrm{m}^{6}$. Crosses represent forward flow, and circle's represent reverse flow. See Figure 7 for definition of forward/reverse flow.

\section{SOLUTIONS FOR FLOW RATE AS A FUNCTION OF IMPELLER ROTATION SPEED}

\subsection{Steady flow conditions}

The first solution we consider is for steady flow. In this case, the rotation speed of the pump will be con- stant and so the first term on the right hand side of (8) vanishes. Consequently, (8) reduces to the solution

$Q=N / \sqrt{a_{2}^{\prime}}$

This result implies that the flow rate will be proportional to the rotation speed of the impeller, and that the coefficient $a_{2}^{\prime}$ can be obtained from the gradient of a plot of measured steady flow rate as a function of pump rotation speed.

To test this relationship, the impeller within the MOT was run at different steady rotation speeds and the resulting flow rate was measured by integrating cross-sectional mean velocity profiles (sampled at 50 $\mathrm{Hz}$ and averaged over 6 minutes; see Section 7 for more details). Figure 2 presents these measurements for flow in both directions (i.e. propeller run in forwards and reverse rotation) and indicates a clear linear relationship between flow rate and pump speed, in agreement with (9). From the gradient of the best fit line $a_{2}^{\prime}=4.14 \times 10^{9} \mathrm{rpm}^{2} \mathrm{~s}^{2} / \mathrm{m}^{6}$ for clockwise and anti-clockwise flow directions. This value is dependant on the geometry of the O-tube (and losses within the pipe etc.) and should, therefore, be a fixed value for a given O-tube and impeller arrangement. Additionally to the empirical approach, one can estimate $a_{2}^{\prime}$ by using equation (6a) accumulating all potential frictional and minor losses due to bends and crosssection changes in the O-tube. A 'broad brush' calculation showed good agreement with measured values based on Figure 2. This consistency gives confidence in Equation (9).

\subsection{Oscillatory flow conditions}

A second analytical solution to (8) can be obtained for oscillatory flow conditions if it is assumed that the non-linear frictional losses are small compared with the acceleration of water in the O-tube, so that the second term on the right hand side of (8) can be neglected. This is most likely to be appropriate for short period oscillatory flow with small peak flow rate. Adopting this assumption the solution to (8) can then be obtained for a sinusoidal variation in rotation speed of $N_{0} \cos (\omega t)$, where $\omega$ is the angular frequency, by setting $|N| N=N^{2}$ over the first quarter cycle and by exploiting even and half wave symmetry in the solution. This leads to the solution over the first period $T(=2 \pi / \omega)$ of

$$
Q(t)= \begin{cases}X(t) & , t \leq \frac{T}{4} \\ X\left(\frac{T}{2}-t\right) & , \frac{T}{4}<t \leq \frac{T}{2} \\ -X\left(t-\frac{T}{2}\right) & , \frac{T}{2}<t \leq \frac{3 T}{4} \\ -X(T-t) & , \frac{3 T}{4}<t \leq T\end{cases}
$$

where 
$X(t)=\operatorname{sign}(\cos (\omega t)) \times \frac{\pi N_{0}^{2}}{4 \omega a_{1}^{\prime}}\left(\frac{2 \omega t}{\pi}+\frac{\sin (2 \omega t)}{\pi}\right)$.

For $t>T$ the flow rate can be obtained by noting that the solution is periodic

(i.e. $Q(t+T)=Q(t)$ ).

To explore the validity of (10), Figure 3 shows an example measurement of flow rate over time recorded in the O-tube. In order to obtain the measured timevarying flow rate over a wave cycle, velocity measurements (sampled in intervals of $20 \mathrm{~ms}$ ) were ensembleaveraged over 50 waves and then integrated over the cross-sectional area (see Section 7 for more details). The input rotation speed was sinusoidal with an amplitude of $N_{0}=875 \mathrm{rpm}$ and a wave period of $10 \mathrm{~s}$. Using $a_{1}^{\prime}=1.18 \times 10^{8} \mathrm{~m}^{3} / \mathrm{rpm}^{2}$ the solution from (10) is in very good agreement with this measurement. The slight discrepancy after the minimum and maximum flow rate is a result of small frictional losses and/or a delay in the impeller response at the zero angular velocity crossing point. In order to show these phenomena, Equation (8) was solved using $a_{2}^{\prime}$ from the previous steady flow condition test (see Section 4.1). Fitting this non-linear prediction to the measurement explains clearly that the faster decrease in flow rate after the amplitude of the wave cycle is due to frictional losses.

From (10) and (11) it is also possible to show that the peak flow rate $Q_{0}$ occurs at $T / 4$ (and every halfperiod following this) and it is equal to

$$
Q_{0}=\frac{\pi N_{0}^{2}}{4 \omega a_{1}^{\prime}} .
$$

Hence, measurement of the peak rotation speed $N_{0}$ and peak flow rate $Q_{0}$ can be used to determine the constant $a_{1}^{\prime}$. We have compared this relationship between the peak impeller speed and peak flow rate with measurements taken in the MOT. Figure 4 presents the measured results for waves (representing an ensemble-average over 50 waves) with varying input rotation speeds $(296 \mathrm{rpm}, 593 \mathrm{rpm}$, $889 \mathrm{rpm}, 1185 \mathrm{rpm}, 1482 \mathrm{rpm})$ and periods $(7.5 \mathrm{~s}$, $10 \mathrm{~s}, 12.5 \mathrm{~s})$. It can be seen that the relationship in (12) (see Figure 4(b)) shows excellent agreement with measurements for sufficiently small peak period (so that the assumptions underlying its derivation are correct) simplifying the calibration of $a_{1}^{\prime}=$ $1.18 \times 10^{8} \mathrm{~m}^{3} / \mathrm{rpm}^{2}$.

Figure 4(a) also includes predictions based on the full form of Equation (8) using $a_{1}^{\prime}$ and $a_{2}^{\prime}$ equivalent to that used in Figure 3. The predicted peak flow rates show good agreement with the measurements across the full range of measurements.

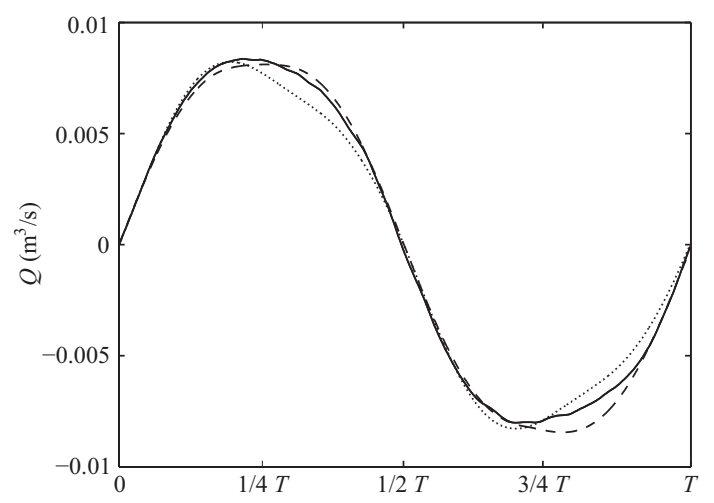

Figure 3: Comparison between wave measurement (solid black line) and two theoretical predictions due to (9) on the one hand neglecting frictional losses (dashed line) and on the other hand considering frictional losses (dotted line). The O-tube specific constants were chosen as $a_{1}^{\prime}=1.18 \times 10^{8} \mathrm{~m}^{3} / \mathrm{rpm}^{2}$ and $a_{2}^{\prime}=$ $4.14 \times 10^{9} \mathrm{rpm}^{2} \mathrm{~s}^{2} / \mathrm{m}^{6}$. The peak rotational speed is $889 \mathrm{rpm}$.

\section{CONTROL OF THE O-TUBE IN COMBINED WAVE AND CURRENT CONDITIONS}

When both steady (current) and oscillatory (wave) pressure is applied via the pump, the form of (8) suggests that the steady and unsteady flow will interact non-linearly (note that this interaction is dependent on the flume and is distinct from non-linear interaction in, for example, the wave and current boundary layer). For these combined flows (8) can be solved numerically, using the values for $a_{1}^{\prime}$ and $a_{2}^{\prime}$ calibrated from steady and oscillatory only conditions, to predict the resulting flow rate. To demonstrate this, Figure 5 presents an input rotation speed having a periodic variation around a non-zero mean speed. Reasonable agreement is shown between the measured flow rate that results from this input rotation speed and the prediction based on (8). The agreement is clearly much better than a prediction based on a simple superposition of the linear component and the periodic component (i.e. simply the addition of the steady flow from (9) and the oscillatory flow from (10) using the same calibration factors for $a_{1}^{\prime}$ and $a_{2}^{\prime}$ ). The superimposed result is a poorer prediction in terms of both phase and magnitude.

\section{VARIATION IN PRESSURE AROUND AN O-TUBE}

So far we have investigated the relationship between impeller rotation speed and flow rate, showing good agreement between theory and measurements in the MOT. This implies that the pressure supplied by the impeller is indeed proportional to the square of its rotation speed (as assumed via the affinity laws). To further confirm this conclusion, Figure 6 presents measurements of the pressure difference across the impeller as a function of steady rotation speed (in this figure measurements were obtained using differential silicon-diaphragm pressure sensors sampling at $50 \mathrm{~Hz}$ ). The results are in excellent agreement with 

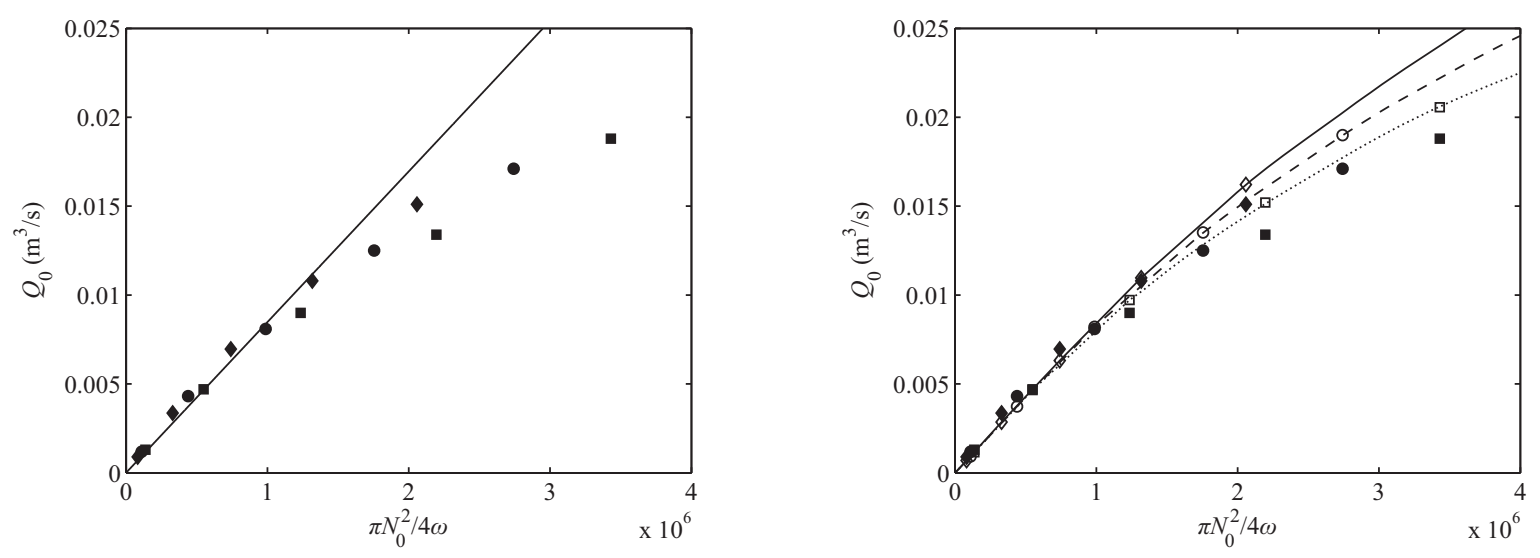

Figure 4: Comparison of peak measured flow rate $Q_{0}$ with the square of the peak input impeller speed $N_{0}$, divided by 4 times the wave frequency divided by $\pi$. The measurements were taken for a wave period of $7.5 \mathrm{~s}$ (filled diamonds), $10.0 \mathrm{~s}$ (circles) and $12.5 \mathrm{~s}$ (squares). (a) The line is an approximate relationship based on (12) with $a_{1}^{\prime}=1.18 \times 10^{8} \mathrm{~m}^{3} / \mathrm{rpm}^{2}$. (b) The full line with diamonds is the fit based on the full Equation (8) for a wave period of $7.5 \mathrm{~s}$, the dashed line with circles for $10 \mathrm{~s}$ and the dotted line with squares for $12.5 \mathrm{~s}$ with $a_{1}^{\prime}=1.18 \times 10^{8} \mathrm{~m}^{3} / \mathrm{rpm}^{2}$ and $a_{2}^{\prime}=4.14 \times 10^{9} \mathrm{rpm}^{2} \mathrm{~s}^{2} / \mathrm{m}^{6}$.

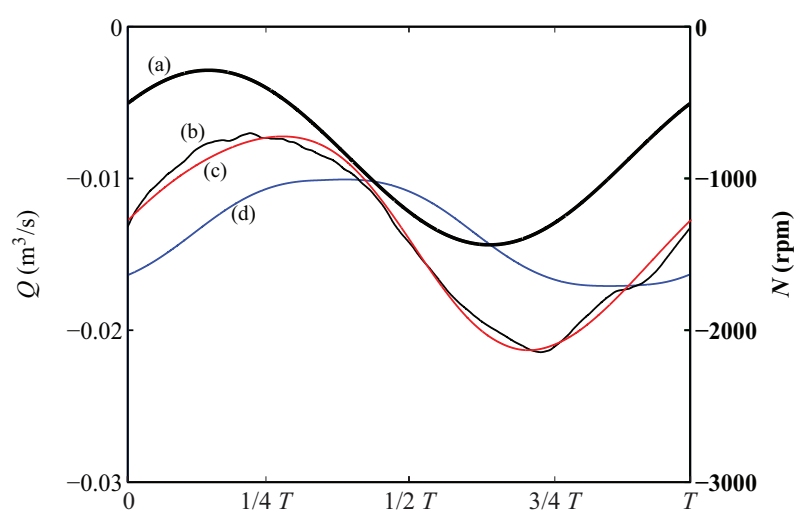

Figure 5: Prediction of flow rate in combined steady and oscillatory conditions. Right axis: line (a) is the impeller rotation speed. Left axis: line (b) is measured data; line (c) is numerical solution of (8) using calibrated values of $a_{1}^{\prime}$ and $a_{2}^{\prime}$; and line (d) is superposition of separate solutions to (9) and (10).

the assumed relationship.

The results in Figure 6 confirm the pressure change at the impeller. Next, we investigate how this supplied pressure varies around the O-tube. Our motivation for investigating this is that, as mentioned in the Introduction, these variations can have important implications for understanding the potential of, for example, seabed liquefaction within the O-tube. To investigate variations in pressure, readings were made at 10 locations for an oscillatory flow $Q_{0}=0.022 \mathrm{~m}^{3} / \mathrm{s}$ with a period of $10 \mathrm{~s}$. Figure 7 presents the measurement results in terms of the amplitude in pressure measured at each location along the O-tube. This amplitude was obtained from an ensemble average of the time varying pressure over 50 waves.

It can be seen in Figure 7 that the amplitude in pressure varies linearly along the tubular and working sections of the O-tube, with a larger gradient in the tubular section where the average velocity is larger (note that a black line is used for flow in the positive $x$ direction and a red line for the reverse direction). It can also be seen that zero pressure occurs within the test

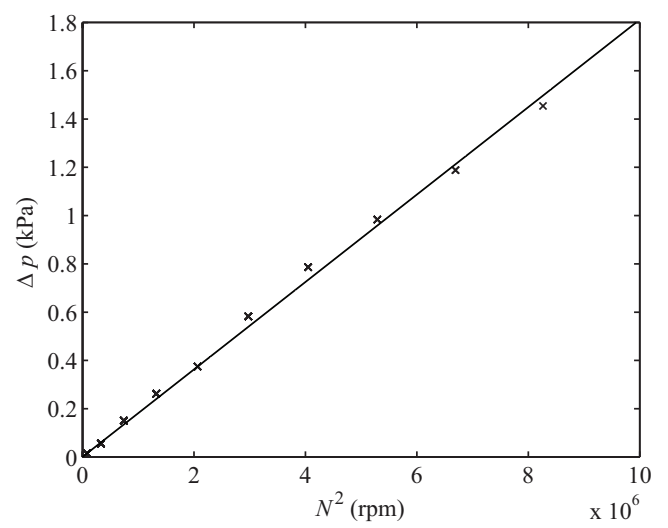

Figure 6: Comparison of measurements of rotation speed against pressure difference across the impeller. Pressure difference is measured at locations $\pm 100 \mathrm{~mm}$ from the impeller. Measurements are taken for the flow in forward direction. See Figure 7 for definition of forward/reverse flow. The calibration factor between pressure and rotation speed yields $a_{3}=1.6 \times$ $10^{-3} \mathrm{~Pa} / \mathrm{rpm}$.

section at $s \sim 5.65 \mathrm{~m}$, which coincides with the centre of water mass either side of the impeller. Each of these results is expected based on the form of Equation (8) if the non-linear drag (the second term on the right hand side) is negligible in comparison to acceleration.

In terms of interpreting the potential for seabed liquefaction in the O-tube, an important observation from Figure 7 is that the pressure distribution within the test section is similar to that expected under the node of a standing wave (assuming linearity through and close to the node) as opposed to the sinusoidal variation in pressure expected for a linear progressive wave. This difference is unavoidable for a water tunnel, in which the free surface cannot be simulated, and implies that the potential for generating momentary liquefaction of a soil within an O-tube may be inhibited. This is because momentary liquefaction requires a large upward pressure gradient in the soil which can only be produced by a significant reduction in water pressure above the seabed. This reduction in pressure 
may occur under the wave trough of a progressive wave (Sumer, Fredsøe, Christensen, \& Lind 1999), but not at the centre of the O-tube working section where there is a node in pressure.

Despite the pressure distribution within the O-tube resembling a standing wave, this does not inhibit the ability for a soil within the O-tube to experience liquefaction due to the build-up of excess pore pressure (see, for example, Sassa and Sekiguchi (2001)). This is because the applied shear stress causing the generation of excess pore pressure is dependent on the spatial pressure gradient (Teh 2003), as opposed to temporal fluctuations in pressure at a fixed point on the seabed. The actual magnitude in the spatial pressure gradient required to cause liquefaction of a given sediment type depends on the particular characteristics of the sediment (De Groot, Bolton, Foray, Meijers, Palmer, Sandven, Sawicki, \& Teh 2006). However, to give an indication, Sumer and Fredsøe (2002) have shown that sandy silt can liquefy when the pressure gradient exceeds $1.1 \mathrm{~Pa} / \mathrm{m}$ (assuming a $1.6 \mathrm{~s}$ period). This pressure gradient can be reached in the MOT/LOT using wave periods in the range of $2.5-5 \mathrm{~s}$ (Table 1).

\section{DETAILED DESCRIPTION OF THE FLOW FIELD IN THE O-TUBE}

To characterise the flow within the O-tube, a series of measurements were performed covering steady and oscillatory flow conditions. Measurements from this campaign are reported in the following subsections. It should be noted that all velocity readings reported herein were made with an Acoustic Doppler Velocimeter (ADV) from Nortek that can recover simultaneous measurements at 35 points distributed at a spatial resolution of $1 \mathrm{~mm}$ in the vertical direction. During the campaign, a smooth false floor resulting in cross-sectional dimensions of the working section to the dimensions $b \times h=0.2 \mathrm{~m} \times 0.2 \mathrm{~m}$ (see Figure 1).

Mean velocities for steady flow conditions were extracted by averaging the recorded velocity timeseries over at least $6 \mathrm{~min}$ (which was found to recover repeatable mean velocities within a few percent of the true mean value). Oscillatory velocities were ensemble-averaged over 50 wave periods (in a similar manner to Jensen et al. (1989) and Sleath (1987)).

All velocity measurements were made over the full vertical range of the ADV (i.e. $35 \mathrm{~mm}$ ) with the closest velocity measurement taken $10 \mathrm{~mm}$ above the test bed. As we will show later, the velocity profile obtained was in very good agreement with theoretical expectations. We present velocity measurements in the remainder of this paper using a right handed coordinate system with the origin at the central bottom of the working section (see Figure 1).

\subsection{Mean velocity under steady flow conditions}

We start by considering the velocity profiles in steady flow. Figure 8 presents contours of the normalised mean streamwise velocity at $x=0$ (i.e. a horizontal cut through the centre of the working section) for steady flow conditions. These measurements were made for two different flow rates, namely (i) $0.008 \mathrm{~m}^{3} / \mathrm{s}$ and (ii) $0.019 \mathrm{~m}^{3} / \mathrm{s}$ as shown in Figure 8 . Within the cross-section the measured flow velocities were restricted to the dashed measuring area indicated in Figure 8 due to the limited access of the ADV. The contour plots were compiled by piecing together velocity profiles obtained at 9 ADV locations in the $y$ direction and $4 \mathrm{ADV}$ locations in the $z$ direction.

As shown in Figure 8, at any flow rate $(Q=$ $0.008 \mathrm{~m}^{3} / \mathrm{s}$ and $Q=0.019 \mathrm{~m}^{3} / \mathrm{s}$ ) a velocity peak forms towards the outer wall of the test section (i.e. $y / b<0$ ) at a height of roughly $z / h>0.6$. The lateral location of the peak velocity (to the left of the centre of the working cross-section) is in agreement with the findings from Rowe (1970), Patankar et al. (1975) or Sudo et al. (1998), who investigated flows with homogeneous inlet conditions around a bend, and is a result of centrifugal forces in the bend of the O-tube which result in an increased pressure (and, therefore, a decrease in velocity) close to the inner wall (An, Luo, Cheng, \& White 2013). This asymmetry in the flow profile is likely to be a common characteristic to all O-tubes and is important to characterise for experimental interpretation.

Measurements at $x=0$ were also made of the secondary flows at each ADV measurement location. Despite the non-uniformity in the flow profiles shown in Figure 8 , these measurements showed that secondary velocities in the working section were smaller than $5 \%$ of the bulk stream-wise velocity at all points in the cross-section. Because these secondary velocities were small, accurate profiles of the secondary flows could not be generated. The limited size of the secondary flows is reassuring, and important to verify given that the flow is driven by an impeller.

\subsection{Mean velocity under oscillatory flow conditions}

Figure 9 shows the amplitude in velocity obtained from ensemble averaged velocities of the oscillatory flow at $x=0$. The particular profile shown in Figure 9 is for oscillatory flow of period $10 \mathrm{~s}$ and $Q_{0}=$ $0.008 \mathrm{~m}^{3} / \mathrm{s}$, generated by a sinusoidal time-varying impeller speed. The results presented in Figure 9 were similar to those obtained for all peak flow velocities with period $\leq 10 \mathrm{~s}$. It is clear from Figure 9 that the velocity profile is much more uniform than in the steady flow experiments, and this agrees well with preliminary results presented in An et al. (2013). The boundary layer tends to start around $z / b=0.2$ and forms a bulge towards the bottom, which is consis- 


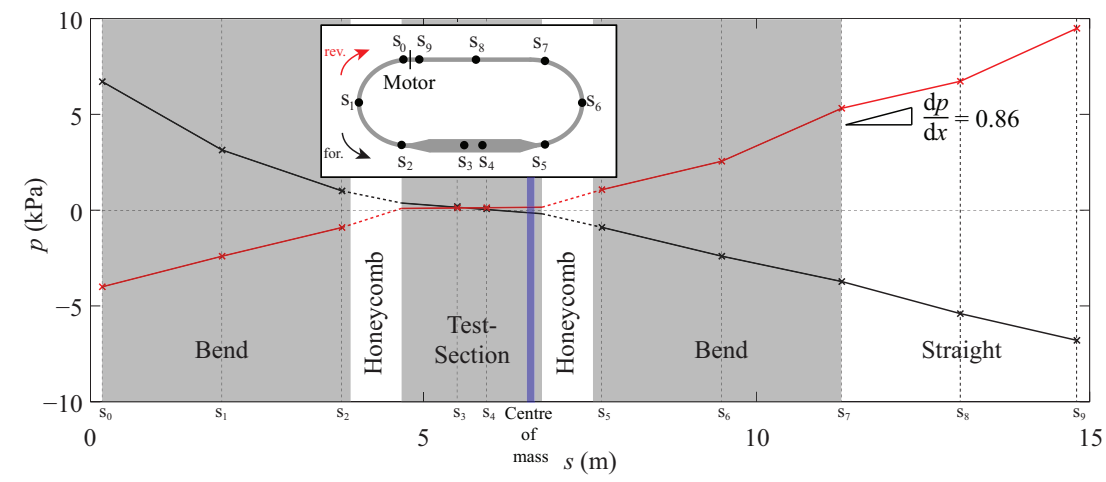

Figure 7: Schematic pressure distribution along the O-tube for a peak flow rate of $0.022 \mathrm{~m}^{3} / \mathrm{s}$ and a period of $10 \mathrm{~s}$. Black line indicates movement in forward direction and a red line in the reverse direction. Parameter, $s$, defines the position around O-tube, as shown in the inset figure.
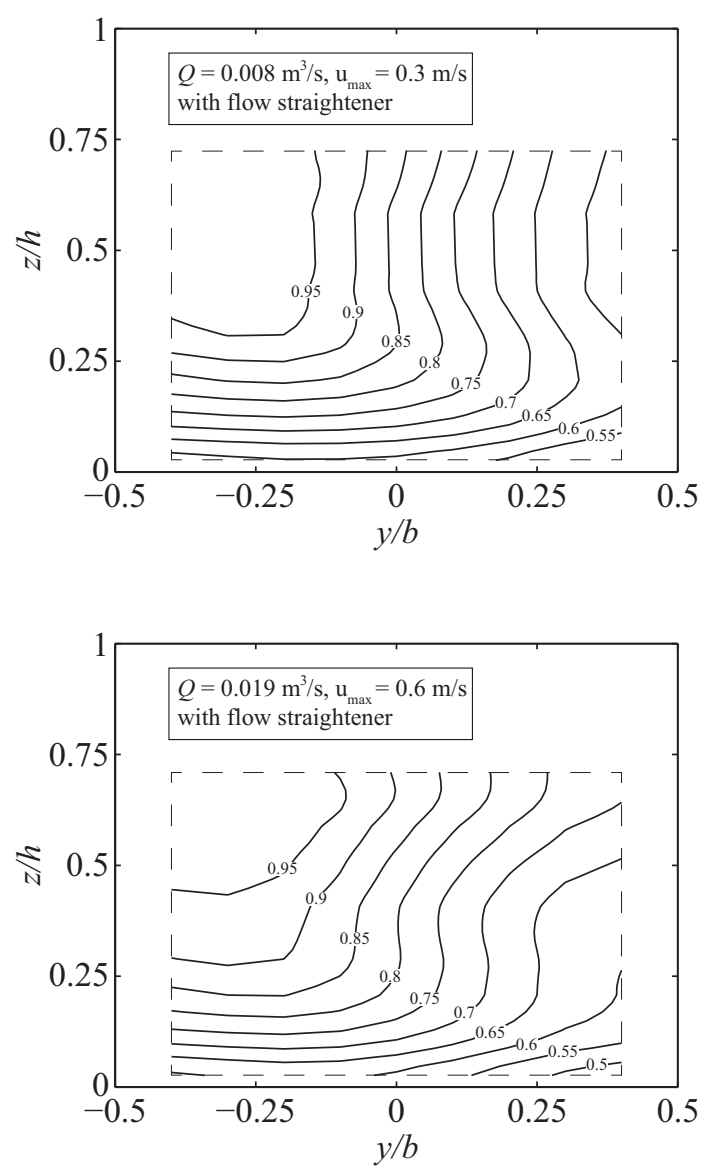

Figure 8: Contours of streamwise velocity normalised by the maximum velocity in the $y z$ plane for two different steady flow rates $\left(Q=0.008 \mathrm{~m}^{3} / \mathrm{s}\right.$ and $\left.Q=0.019 \mathrm{~m}^{3} / \mathrm{s}\right)$ measured at the centre of the working section $(x=0 \mathrm{~mm})$.

tent with that observed by Jensen et al. (1989). The boundary layer thickness increases only slightly towards the outer wall. These results are suggestive of a well-defined velocity profile within the O-tube for oscillatory flow conditions.

\section{LOGARITHMIC PROFILES AND SHEAR STRESS}

Finally to better interpret the velocity profiles presented in Section 7.1, we have also examined the

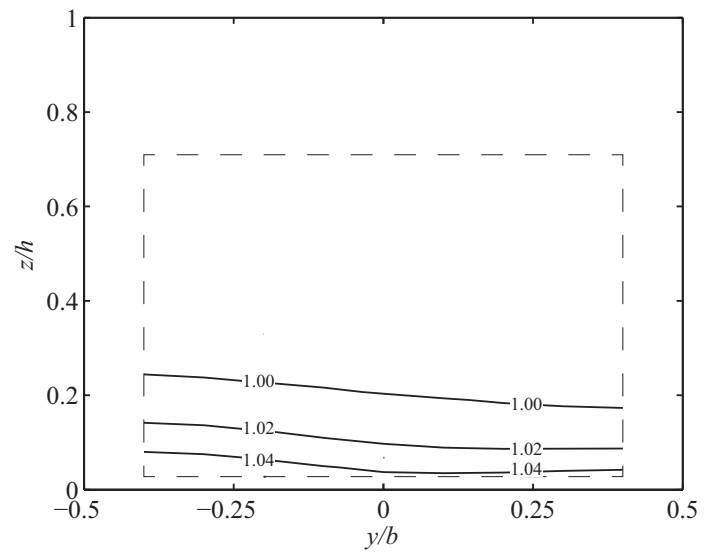

Figure 9: Contours of the amplitude in streamwise velocity normalised by the peak velocity in the $y z$ plane at $x=0$ for an oscillatory flow. This flow condition has a peak flow rate of $0.008 \mathrm{~m}^{3} / \mathrm{s}$, a peak velocity of $0.2 \mathrm{~m} / \mathrm{s}$ and a period of $10 \mathrm{~s}$.

vertical velocity profile of the streamwise velocity in the test section. Firstly, the vertical velocity is compared with logarithmic profiles providing estimations of shear stress calculations. Secondly, shear stress variations are shown for (i) different seabed condition at the centre of the test section and (ii) different locations along the test section showing a developed profile.

Measurements of 22 different steady flow rates with three seabed types: a smooth bed, and two rough sandy beds (prepared by gluing uniform sand with a median grain size of $0.19 \mathrm{~mm}$ and $0.54 \mathrm{~mm}$, respectively, to the surface of the false floor.) Figure 10 presents all of the velocity profiles for these different flow rates and test bed conditions.

For the smooth test bed measurements, Figure 10 shows that all the velocity profiles agree well with Clauser's equation (Clauser (1956))

$u^{+}=(1 / 0.41) \ln \left(z^{+}\right)+4.9$,

where $z^{+}=z u_{*} / \nu$ and $u^{+}=u / u_{*}$. Here $z^{+}$is the wall coordinate, made dimensionless with the friction velocity $u_{*}$ and the kinematic viscosity $\nu . u^{+}$is the dimensionless velocity which is the velocity in the $x$ direction as a function of $z$, divided by the friction ve- 


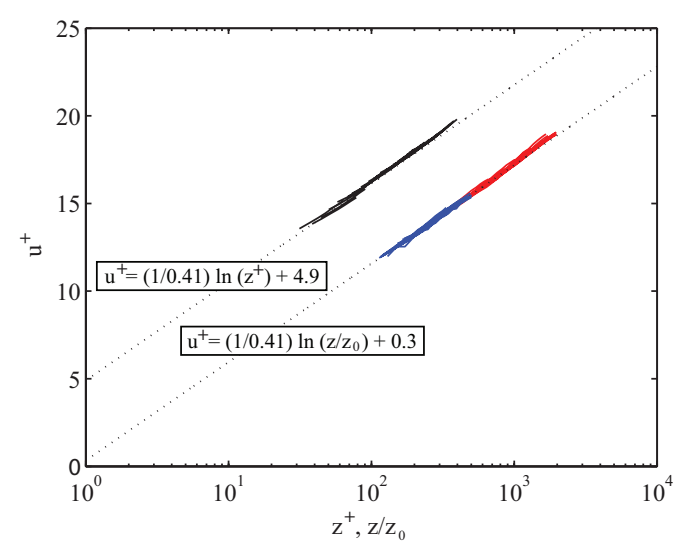

Figure 10: Stream wise velocity profiles measured on a smooth surface (black), and rough surfaces with uniform sand having grain diameter of $0.19 \mathrm{~mm}$ (red) and $0.54 \mathrm{~mm}$ (blue), respectively. The data for the smooth surface is compared with an approximation by Kline et al. (1967) with horizontal coordinate $z^{+}$, whereas the sand surfaces are approximated by a best fit with horizontal coordinate $z / z_{0}$.

locity. For each velocity profile shown in Figure 10, the seabed roughness length was calculated using the empirical fit of Christoffersen and Jonsson (1985) to the results of Nikuradse (see Nikuradse (1933)), together with the friction velocity obtained assuming a logarithmic velocity profile and the measured velocity at a distance $6 \mathrm{~mm}$ from the seabed.

The good agreement in Figure 10 underlines the assumption of Clauser (1956) that the wall flow is not influenced by the turbulent free stream flow (presented in Section 7.1). It also implies that the bed shear stress can be calculated from velocity measurements made within the logarithmic region near the bed of the O-tube.

For the velocity measurements made using the two rough surfaces, Figure 10 illustrates that a logarithmic velocity distribution is again appropriate. The best fit relationship for both bed roughnesses in Figure 10 was found to be

$u^{+}=(1 / 0.41) \ln \left(z / z_{0}\right)+0.3$,

where $z / z_{0}$ is the distance $z$ to the wall divided by the seabed roughness length $z_{0}$.

A key metric in sediment transport is the shear stress at the seabed. As a final exercise, the shear stress for the smooth and rough bed cases were, therefore, also calculated for each flow conditions using two different approaches. Firstly, the friction velocity was obtained by fitting a logarithmic profile of the form $u=u_{*} / 0.41 \ln \left(z / z_{0}\right)$ to each velocity profile using a series of point measurements between $6 \mathrm{~mm}$ and $12 \mathrm{~mm}$ above the seabed. The shear stress was then calculated according to $\rho u_{*}^{2}$. Secondly, the velocity at $6 \mathrm{~mm}$ above the seabed was used to calculate the friction velocity assuming a logarithmic velocity distribution and an estimate for $z_{0}$ based on Christoffersen and Jonsson (1985).

A comparison of the computed shear stress using both approaches across a range of mean flow rates is given in Figure 11(a). It is evident from this figure that both methods give very similar results (to within $4 \%$ for shear stress across all seabed types and flow rates). Therefore, either method is adequate to calculate the shear stress acting on the bed within the working section of the MOT (i.e. a fit to all velocity measurements or an empirical estimate based on one point measurement).

Additional to the variation in shear stress due to changing bed surfaces, measurements were performed to quantify the change in shear stress along the test bed in the working section. Figure 11(b) shows only a minimal change in shear stress $200 \mathrm{~mm}$ before and $200 \mathrm{~mm}$ after the centre of the test section. This leads to the conclusion that the velocity profile is fully developed at the position $x=-200 \mathrm{~mm}$ and hence does not change over the next $400 \mathrm{~mm}$. The shear stress can be assumed as nearly constant over this part of the test section simplifying the interpretation for any test that might be performed in this region of the test section. Details on applications in the MOT are shown in the following section.

\section{EXAMPLE APPLICATIONS}

\subsection{Non-cohesive threshold shear stress}

In any flume it is important to confirm that results for key properties, such as sediment threshold shear stress, are consistent with experimental results in the literature. To ensure this is the case in the MOT, the erosion properties of a number of uniform sediments have been tested. Specifically, measurements of threshold velocity were conducted by placing sediment into a sample holder that resembled one half (cut lengthwise) of a circular cylinder with a diameter of $76 \mathrm{~mm}$ and a length of $\sim 200 \mathrm{~mm}$ (see Figure 12). The sample was then placed within the centre of the MOT working section (so that variations in velocity are small across and along the sample). Steady currents were then introduced in a stepwise fashion until significant erosion was observed. Erosion was quantified via scanning the surface of the sample periodically with a 3-D laser scanner (see, for example, Mohr et al. (2013)). The corresponding threshold shear stress was then back calculated from measurement of the velocity profile over the sample.

Results for loose uniform sediment (ranging from $15 \mu \mathrm{m}$ limestone to $540 \mu \mathrm{m}$ siliceous sand) are given in Figure 12 and compared with the well-known Shields curve (Shields (1936); refitted by Soulsby and Whitehouse (1997)) and laboratory data compiled by Soulsby and Whitehouse (1997). Good agreement is observed across the range of grain sizes, and this gives confidence in using the O-tube for erosion testing.

Preliminary testing results obtained for others sediments, including calcareous sediments (ranging from silty SAND to sandy SILT), are reported in Mohr et al. (2013). 


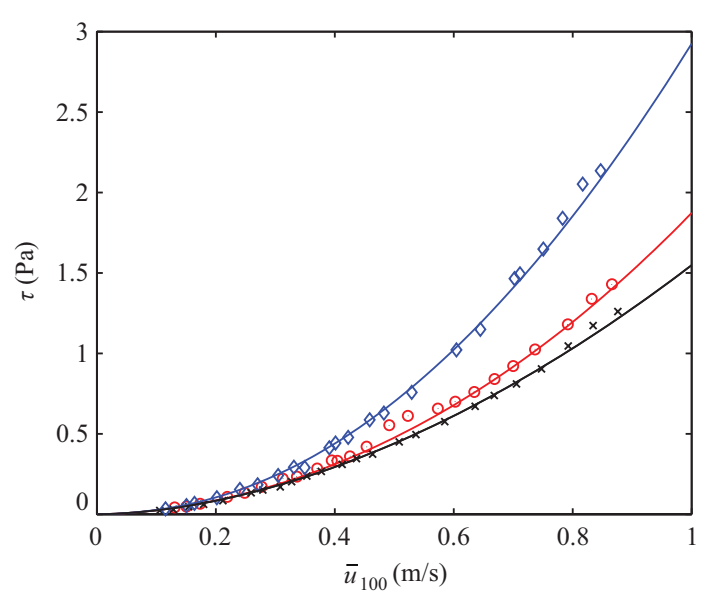

(a) Shear stresses for smooth bed (crosses), a sand with grain diameter $0.19 \mathrm{~mm}$ (circles) and a sand with grain diameter $0.54 \mathrm{~mm}$ (diamonds).

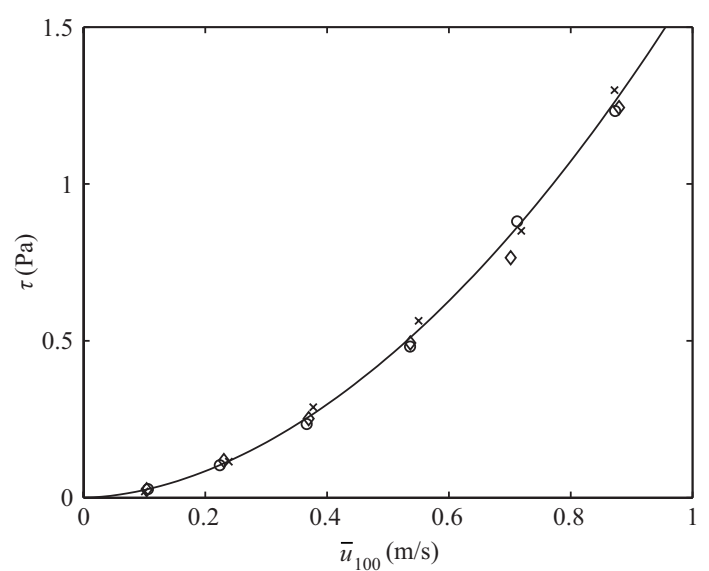

(b) Shear stress for a smooth bed at $x=$ $-200 \mathrm{~mm}$ (circles), at $x=0 \mathrm{~mm}$ (crosses) and $x=200 \mathrm{~mm}$ (diamonds)

Figure 11: Calculated shear stresses using a best least squares fit of the logarithmic profile between $6 \mathrm{~mm}$ and $12 \mathrm{~mm}$ above the seabed. Comparative shear stresses computed using a roughness length derived empirically for a smooth test bed (as outlined in the text) is shown as a continuous line.

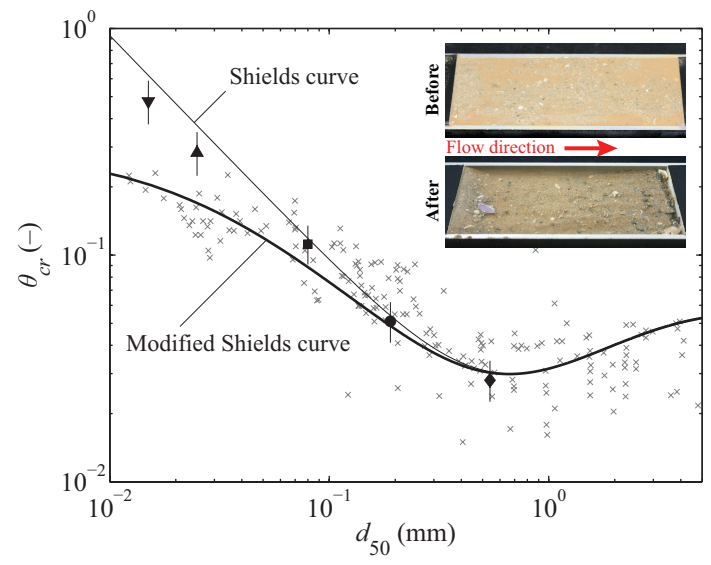

Figure 12: Dimensionless threshold shear stress for uniform sediments with error bar. Also shown are two Shields curves after Soulsby (1997) and empirical results compiled by (Soulsby $\&$ Whitehouse 1997) given as crosses. Average grain size (and uniformity index) of samples are: $15 \mu \mathrm{m}$ (18); $25 \mu \mathrm{m}(30)$; $80 \mu \mathrm{m}$ (2.5); $190 \mu \mathrm{m}$ (1.7); $540 \mu \mathrm{m}$ (1.4).

\subsection{Pipeline scour}

The MOT has also been used to study scour under model pipelines (Figure 9.2). Recent work has considered the critical point at which scour initiates (i.e. the onset of scour) in steady currents (Zhang, Draper, Cheng, An, \& Shi 2013) and the critical point at which scour initiates in combined wave and currents (Zang, Cheng, \& Zhao 2010). These studies have demonstrated good agreement with published measurements of the onset of scour beneath pipelines, equilibrium scour depths in wave and current only conditions and time-scale of scour development. A comparison of MOT results with published results for the onset of scour in steady currents is given in Figure 9.2.

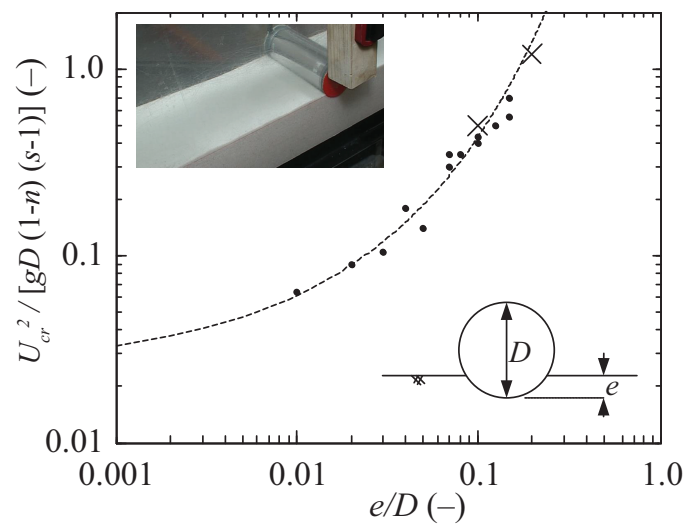

Figure 13: (a) A $50 \mathrm{~mm}$ model pipeline placed rigidly within the MOT working section at a nominal initial embedment into the sand. (b) Non-dimensional parameter $U_{c r} / g D(1-n)(s-1)$ (see Zhang et al. (2013) for further details) describing onset of tunnel scour in steady currents as a function of normalised pipeline embedment $e / D$. Experimental results from (Sumer \& Fredsøe 2002) shown as dots with trend line. Results obtained with the MOT by (Zhang, Draper, Cheng, An, \& Shi 2013) shown as crosses.

\section{CONCLUSIONS}

An O-tube is a recirculating flume that can be used to simulate wave and currents representative of ambient to cyclonic field conditions at the seabed. A key advantage of an O-tube driven by an impeller is that it can reproduce oscillatory flow with peak velocity limited only by the power/efficiency of the impeller; and this ensures that high velocity current and oscillatory flow can be reproduced. However, like all enclosed flumes an O-tube is limited in that it does not reproduce the same orbital wave kinematics or the same relationship between pressure and velocity at the seabed. In this paper, the actual relationship between pressure and flow rate in an impeller driven $\mathrm{O}-$ tube has been derived in terms of a dynamic equation 
extending classical work for flow in closed conduits such as U-tubes (Streeter 1958). Analytical solutions have been given relating the impeller rotation speed to the flow rate in steady and oscillatory flow, respectively. Calibration in these conditions has been shown to allow for optimum control of flow rate in combined wave and current conditions.

Based on the relationship between pressure and flow rate derived in this paper, interpretation of physical phenomena in the O-tube can be improved. For example, by measuring the pressure variations along the O-tube, which are consistent with the dynamic equation, it has been shown that the pressure variation within the working section of an O-tube is similar to that under a node of standing wave. Consequently, seabed liquefaction may be simulated in an O-tube, since the spatial pressure gradient that leads to cyclic shear stresses within the seabed is reproduced.

Finally, detailed measurements of steady and oscillatory flow have been given to characterise the mean flow, flow asymmetry and bed shear stress with the working section of an O-tube. This has documented the cross-sectional velocity profile in the $\mathrm{O}$ tube, which is likely to be similar for all O-tube flumes of similar geometry.

\section{ACKNOWLEDGEMENTS}

This research forms part of the activities of the Centre of Offshore Foundation Systems (COFS), supported as a node of the Australian Research Council's Centre of Excellence for Geotechnical Science and Engineering (CGSE), and through the Fugro Chair in Geotechnics, the Lloyd's Register Foundation Chair and Centre of Excellence in Offshore Foundations and the Shell EMI Chair in Offshore Engineering. The first author acknowledges his Research Studentship support from The University of Western Australia. The first and second author acknowledge the support of the Lloyd's Register Foundation. The Foundation helps to protect life and property by supporting engineering-related education, public engagement and the application of research. The fourth author acknowledges the support of Shell, via the Shell EMI Chair. This research is supported through ARC Discovery Grants Program: DP130104535.

\section{REFERENCES}

Ahmed, S. M. A. (2002). Sheet flow transport mechanism of heterogeneous sediments under nonlinear oscillatory flows. $\mathrm{Ph}$. D. thesis, University of Tokyo.

An, H., C. Luo, L. Cheng, \& D. White (2013). A new facility for studying ocean-structure-seabed interactions: The Otube. Coastal Engineering 82, 88-101.

Bagnold, R. A. \& G. Taylor (1946). Motion of waves in shallow water. Interaction between waves and sand bottoms. Proc. of the Royal Society of London. Series A, Mathematical and Physical Sciences 187(1008), 1-18.
Biery, J. C. (1969). The oscillating manometer: A review of experimental, theoretical, and computational results. American Institute of Chemical Engineers Journal 15(4), 631-634.

Cheng, L., D. White, A. Palmer, E. Jas, A. Czajko, A. Fogliani, R. Fricke, \& H. An (2010). A new facility for research on the stability of pipelines on unstable seabeds. In Offshore Pipeline Technology Conference.

Christoffersen, J. B. \& I. Jonsson (1985). Bed friction and dissipation in a combined current and wave motion. Ocean Engineering 12(5), 387-423.

Clauser, F. H. (1956). The turbulent boundary layer. Advances in Applied Mechanics 4, 1-51.

Davies, A. G. \& R. G. Wilkinson (1978). Sediment motion caused by surface water waves. Coastal Engineering Proceedings 1(16), 1577-1595.

De Groot, M. B., M. D. Bolton, P. Foray, P. Meijers, A. C. Palmer, R. Sandven, A. Sawicki, \& T. C. Teh (2006). Physics of liquefaction phenomena around marine structures. Journal of Waterway, Port, Coastal, and Ocean Engineering 132(4), 227-243.

Hammond, T. M. \& M. B. Collins (1979). The design and use of an oscillating trolley wave-simulator, for installation in unidirectional flow flumes. Estuarine and Coastal Marine Science 9(6), 801-811.

Jensen, B. L., B. M. Sumer, \& J. Fredsøe (1989). Turbulent oscillatory boundary layers at high reynolds numbers. Journal of Fluid Mechanics 206, 265-297.

Kline, S. J., W. C. Reynolds, F. A. Schraub, \& P. W. Runstadler (1967). The structure of turbulent boundary layers. Journal of Fluid Mechanics 30(04), 741-773.

Mohr, H., S. Draper, \& D. J. White (2013). Free field sediment mobility on Australia's North West Shelf. In Proc. 32nd International Conference on Ocean, Offshore and Arctic Engineering, pp. V04BT04A051, 11 pages.

Moody, L. F. (1944). Friction factors for pipe flow. Transactions of the American Society of Mechanical Engineers 66(8), 671-684.

Murray, P. B. (1992). Sediment pick-up in combined wavecurrent flow. Ph. D. thesis, University College of North Wales.

Nikuradse, J. (1933). Laws of flow in rough pipes. National Advisory Committee for Aeronautics, Washington D.C.

O'Donoghue, T. \& S. Wright (2004). Flow tunnel measurements of velocities and sand flux in oscillatory sheet flow for wellsorted and graded sands. Coastal Engineering 51(11), 11631184.

Patankar, S. V., V. S. Pratap, \& D. B. Spalding (1975). Prediction of turbulent flow in curved pipes. Journal of Fluid Mechanics 67(3), 583-595.

Ribberink, J. S. \& A. A. Al-Salem (1994). Sediment transport in oscillatory boundary layers in cases of rippled beds and sheet flow. Journal of Geophysical Research: Oceans 99(C6), 12707-12727.

Richardson, P. D. (1967). Free oscillations of fluids in manometers. American Institute of Chemical Engineers Journal 13(4), 821-823.

Rowe, M. (1970). Measurements and computations of flow in pipe bends. Journal of Fluid Mechanics 43(04), 771-783.

Sassa, S. \& H. Sekiguchi (2001). Analysis of wave-induced liquefaction of sand beds. Geotechnique 51(2), 115-126.

Shields, A. (1936). Anwendung der Aehnlichkeitsmechanik und der Turbulenzforschung auf die Geschiebebewegung. Ph. D. thesis, Preußiche Versuchsanstalt für Wasserbau und Schiffbau.

Sleath, J. F. A. (1987). Turbulent oscillatory flow over rough beds. Journal of Fluid Mechanics 182, 369-409.

Soulsby, R. L. (1997). Dynamics of marine sands: A manual for practical applications. Thomas Telford, London.

Soulsby, R. L. \& R. J. S. Whitehouse (1997). Threshold of sediment motion in coastal environments. In Proc. 13th Australasian Coastal and Ocean Engineering Conference and 
6th Australasian Port and Harbour Conference, Volume 1, pp. 145.

Streeter, V. (1958). Fluid Mechanics. McGraw-Hill, New York.

Sudo, K., M. Sumida, \& H. Hibara (1998). Experimental investigation on turbulent flow in a circular-sectioned 90-degree bend. Experiments in Fluids 25(1), 42-49.

Sumer, B. M. \& J. Fredsøe (2002). The mechanics of scour in the marine environment. World Scientific Publishing, Singapore.

Sumer, B. M., J. Fredsøe, S. Christensen, \& M. T. Lind (1999). Sinking/floatation of pipelines and other objects in liquefied soil under waves. Coastal Engineering 38(2), 53-90.

Teh, T. C. (2003). Stability of marine pipelines on unstable and liquefied seabed. $\mathrm{Ph}$. D. thesis, University of Cambridge.

Zang, Z., L. Cheng, \& M. Zhao (2010). Onset of scour below pipeline under combined waves and current. In Proc. 29th International Conference on Ocean, Offshore and Arctic Engineering, pp. 483-488.

Zhang, Q., S. Draper, L. Cheng, H. An, \& H. Shi (2013). Revisiting the mechanics of onset of scour below subsea pipelines in steady currents. In Proc. 32nd International Conference on Ocean, Offshore and Arctic Engineering, pp. V04BT04A039; 11 pages. 